\title{
Efficacy and Safety of A Novel Antiviral Preparation in ICU-Admitted Patients with COVID-19: A Phase III Randomized Controlled Trial
}

\author{
Hossein Faramarzi \\ Shiraz University of Medical Sciences \\ Amirhossein Sahebkar \\ Mashhad University of Medical Sciences \\ AHMAD HOSSEINPOUR ( $\square$ ahosseinpour3@gmail.com ) \\ Shimi Teb Salamat Co \\ Vahid Khaloo \\ Shiraz University of Medical Sciences \\ Parisa Chamanpara \\ Shiraz University of Medical Sciences \\ Mohammad Reza Heydari \\ Shiraz University of Medical Sciences \\ Sajad Najafi \\ Shahid Beheshti University of Medical Sciences \\ Fatemeh Fotoohi Khankahdany \\ Shimi Teb Salamat Co \\ Ahmad Movahedpour \\ Behbahan Faculty of Medical Sciences
}

Research Article

Keywords: Coronavirus, SARS-CoV-2, COVID-19, Treatment, ICU Hospitalization, Mortality Rate

Posted Date: February 7th, 2022

DOI: https://doi.org/10.21203/rs.3.rs-1318760/v1

License: @ (1) This work is licensed under a Creative Commons Attribution 4.0 International License. Read Full License 


\section{Abstract}

Introduction

Despite an increasing number of studies, there is as yet no efficient antiviral treatment developed for COVID-19. In this clinical trial, we examined the efficacy of a novel herbal antiviral preparation comprising Zataria multiflora Boiss, Glycyrrhiza glabra, Cinnamomum Vermont, Allium sativuml, and Syzygium aromaticum in critically ill patients with COVID-19.

\section{Methods}

A total number of 120 ICU-admitted patients requiring pulmonary support with a diagnosis of COVID-19 pneumonia were recruited to the trial. Participants were equally randomized to receive either the novel antiviral preparation sublingually, for up to two consecutive weeks or till discharge, or placebo. Clinical and laboratory parameters as well as survival rates were compared between the two groups at the study end.

\section{Results}

The cumulative incidence of death throughout the study period was $8.33 \%$ in the intervention group and $60 \%$ in the placebo group (risk ratio: $0.14 ; 95 \%$ confidence interval [Cl], 0.05 to $0.32 ; \mathrm{P}<0.001$ ). Survival rates were significantly higher in the intervention group. Additionally, on day 7 , several laboratory factors including white blood cells (WBCs) count, C-reactive protein, and $\mathrm{SpO}_{2}$ were improved in patients treated with the novel antiviral preparation compared with the placebo group.

\section{Conclusion}

The novel antiviral preparation tested in this trial significantly improved the survival rate and reduced mortality in critically ill patients with COVID-19. Thus, this preparation might be suggested as a potentially promising COVID-19 treatment.

Funded by Shimi Teb Salamat Co., Shiraz, Iran, and registered on the Iranian registry of clinical trials (Trial registration: IRCT20200509047373N2. Registered 13 April 2021).

\section{Introduction}

The first cases of novel coronavirus disease (COVID-19) pandemic were initially reported in Wuhan city in the Hubei province of China during late December 2019 (1). By November 5, 2021, nearly a quarter billion people have been identified to be infected with the causative virus SARS-CoV-2 globally and the total worldwide death toll has exceeded 5 million (2). SARS-CoV-2 is an RNA virus, which attaches to the angiotensin-converting enzyme 2 (ACE2) receptor on the target cells via its spike (S) protein. Following entry into the host cells, the virus is fused to endosomes and uncoated. Then, primary proteins are translated and viral single-stranded RNA (ssRNA) genome is replicated. At the last steps, virions are assembled and released from the infected cells (3). SARS-CoV-2 is known to be highly transmitted through respiratory fluids including droplets and aerosol particles with higher transmission rates in asymptomatic infected individuals, which has made the viral infection more prevalent and control of the disease more challenging (4). COVID-19 also demonstrates higher morbidity among affected patients with a broader age range and despite lower proportion of pediatric patients, severe disease has been reported in COVID-19-affected children (5). The majority of COVID-19 patients recover from the disease without receiving medical care or hospitalization and receiving supportive treatments, while a proportion develop severe grades of the disease requiring mechanical ventilation and hospitalization in the intensive care unit (ICU). The highest mortality rates among COVID-19 cases have been reported in these critically ill patients by as high as $50 \%$ to $67 \%(6,7)$. These patients develop severe complications of COVID-19 like acute respiratory distress syndrome (ARDS), which along with extrapulmonary conditions are responsible for poor outcomes (8). Prolonged ICU hospitalization and invasive mechanical ventilation cause pulmonary infections leading to septic shock and multi-organ failure as the immediate contributing conditions to the majority of deaths (9). Thus, management of ICU-admitted patients has faced numerous challenges since the pandemic emergence.

Globally, after development of highly efficient vaccines for SARS-CoV-2, mass vaccination has greatly helped preventing severe COVID-19 cases, hospital and ICU admissions, and total mortalities (10-13). Theoretically, several steps of viral life cycle and SARS-CoV-2-encoded proteins (e.g., viral proteases) could be inhibited to block the virus pathogenesis. However, despite a large number of studies, an efficient prophylactic or therapeutic regimen is yet to be discovered. Although several drugs such as corticosteroids, hydroxychloroquine lopinavir-ritonavir and remdesivir have been suggested as potential treatments, the findings of randomized controlled trials have been equivocal or suggested futility of some of these agents on the recovery of COVID-19 patients or decreasing the mortality rates (14-16). Considering the paucity of effective therapeutic agents, especially in critically ill patients, this study examined the efficacy of a herbal antiviral preparation on clinical symptoms, paraclinical parameters, and survival rates of ICU-admitted patients in a phase III clinical trial. This novel preparation is composed of several herbal ingredients including Zataria multifloraBoiss, Glycyrrhiza glabra, Cinnamomum Vermont, Allium sativuml, and Syzygium aromaticum extracts. The preparation has previously shown antiviral activity in cell experiments and the current study is the first clinical experiment to investigate its efficacy in patients with COVID-19. Since mortality among ICU-admitted patients is significantly higher due to underlying conditions and medications, we set out to evaluate the efficacy of this antiviral preparation in critically ill COVID-19 patients. The results of this study can be extended to outpatients, who have less pulmonary involvement and less co-morbidities and mortality.

\section{Methods}

\section{Trial Design and Participants}


COVID-19 patients hospitalized in an ICU department of Shiraz University of Medical Sciences, Shiraz, Iran, were enrolled in a randomized, double-blind, placebo-controlled phase III clinical trial conducted during March 21- June 19,2021. The trial was registered on the Iranian registry of clinical trials (https://www.irct.ir) (Trial registration: IRCT20200509047373N2. Registered 13 April 2021).The trial site was at Ali Asghar hospital, as one of the ICU centers specialized for caring COVID-19 patients in Shiraz city, to enroll the eligible subjects. ICU-admitted patients were included in the trial with several eligibility criteria including having confirmed diagnosis of COVID-19, high pulmonary involvement in imaging, requirement to respiratory support but not mechanical ventilation (however, they may require mechanical ventilation during trial), aged 15 years or older, filled informed consent, and not participated in other clinical trials. Pregnant women and patients younger than 15 years were excluded from the study. COVID-19 definite diagnosis was made by the infectious diseases specialist according to the national COVID-19 committee's guidelines for diagnosis and management of COVID-19 patients via SARS-CoV-2 reverse transcriptase polymerase chain reaction (RT-PCR) positive results or through characteristic radiographic presentations in chest computed tomography (CT) or radiographic imaging. Written informed consent was obtained from all patients or their legal representatives. The research was approved by the Ethics Committee of the Shiraz University of Medical Sciences (registration No. IR.SUMS.REC.1399.1367). Eligible patients were randomly assigned in a 1:1 ratio to either medication or placebo group.

\section{Sample Size}

No prior data was available on the efficacy of the antiviral preparation in the management of COVID-19, so we performed a pilot study with 30 participants (15 per group) with the same protocol on similar patients and recorded the death rate as the preliminary data on the clinical efficacy of the preparation. According to the results, three $(20 \%)$ of the participants in the antiviral preparation group and seven $(46.7 \%)$ in the placebo group had an event (death through day 14$)$. By considering $90 \%$ power to detect a between-group difference and the alpha level of $5 \%$, we estimated a population size of 120 patients (60 per group).

\section{Randomization and Interventions}

Randomization was conducted by the statistical analyst using the website Randomization.com (http://www.randomization.com) and the permuted block randomization method with a block size of 4 . Patients were enrolled by a health worker rather than the research team and the statistical analyst performed the patient allocation. Allocation was masked to all individuals including patients, physicians and statistical analysts. Both groups received CoVID-19 local standard treatments including remdesivir and glucocorticoids according to the guidelines. Those patients with the underlying conditions also received their medications according to their past prescriptions. Patients assigned to the medication group received the antiviral preparation. Each patient was administered $1 \mathrm{ml}$ (containing $433 \mathrm{mg}$ of active materials) antiviral preparation in drop form using dropper every 3 hours for up to two consecutive weeks. Moreover, patients who underwent mechanical ventilation received the medicine up to 14 days or till discharge. Subjects in the placebo group were given a matching placebo containing normal saline in the same packaging with similar appearance, volume, and dosing as the active compound to maintain blinding. Standard care was provided for all patients in both groups.

\section{Antiviral preparation Composition and dosing}

The novel antiviral preparation tested in this study has already been identified with antiviral properties in cell culture experiments (data not published), and comprised several active herbal ingredients. Plant materials included Zataria multiflora Boiss (5mg), Glycyrrhiza glabra (10mg), Cinnamomum Vermont (2mg), Allium sativum ( $2 \mathrm{mg})$, and Syzygium romaticum (1 $\mathrm{mg})$. Plant materials were purchased from a local botanical supplier in Iran, and were then approved by a plant classification specialist in the Department of Botany, Shiraz University of Medical Sciences, Shiraz, Iran. All plant materials were cleaned, dried, mechanically powdered, and extracted with $100 \%$ deionized water. The water was then evaporated using hot plate at $270^{\circ} \mathrm{C}$ to prepare the extract, followed by filteration. Plant material powders were mixed separately in $200 \mathrm{ml}$ of distilled water. These suspensions were filtered by high-altitude centrifuge and then mixed using a magnetic stirrer. The final extract was placed in the refrigerator until it was ready for use. The extract was used in drops at a final concentration of $433 \pm 5 \mathrm{mg} / \mathrm{ml}$ to be used sublingually. The sterility testing was performed to check the bacterial contamination in each extract. For sterility testing, nutrient agar plates were used and $0.1 \mathrm{~mL}$ of each extract was poured on it and incubated at $37^{\circ} \mathrm{C}$. The bacterial growth was observed after 24 hrs. Reverse phase chromatographic analyses were carried out under gradient conditions using $\mathrm{C} 18$ column $(4.6 \mathrm{~mm} \times 250 \mathrm{~mm})$ packed with $5 \mu \mathrm{m}$ diameter particles. The extract was dissolved in HPLC grade solvent. Then, the sample was sonicated using ultrasonicator for $10 \mathrm{~min}$. The extract was filtered and injected to the HPLC column using a mobile phase comprising acetonitrile: $0.1 \%$ phosphoric acid micture in a $30: 70$ ratio (v/v). The preparation was standardized based on 5.82 mg glycyrrhizic acid, and $2.5 \mu \mathrm{g}$ cinnamaldehyde per each gram of the final preparation. The above procedures were undertaken in the Shimi Teb Salamat Co., Shiraz, Iran.

\section{Procedures}

Throughout the trial since days 0 to 14, patients were assessed for their clinical status. Any adverse effect or exacerbation in outcomes was recorded. Demographic characteristics, age, sex, BMI, and underlying diseases were collected at baseline. Several clinical and paraclinical parameters were evaluated in both groups on day 0 , and for those survived in days 7 and 14. Vital signs including body temperature, blood pressure, respiratory rate, and blood oxygen saturation $\left(\mathrm{SpO}_{2}\right)$, laboratory parameters such as hemoglobin $(\mathrm{Hb})$ values, white blood cells $(\mathrm{WBCs})$ count, and C-reactive protein (CRP) levels were measured. SARS-CoV RT-PCR was conducted at the first time point via standard mid-nasal sterile swab samples. Pulmonary involvement was investigated via chest CTscans. Co-existing conditions were also compared between medication and placebo groups. No significant change was applied in the final protocol compared to the initial planned protocol.

\section{Outcomes}

The main primary outcome was the COVID-19-associated deaths, which was assessed through day 14 after enrollment. Other outcomes were clinical status, and changes in vital signs and laboratory parameters. Any possible adverse effect causing discontinuation of the study and impacting progression of the 
disease, as well as clinical or laboratory parameters were all recorded. No change was applied in the outcomes after trial was commenced.

\section{Statistical Analysis}

The retrieved data was exported to SPSS software, version 18.0 (SPSS Statistics). To compare the qualitative and quantitative variables, Chi-square, independent samples t-test, paired samples t-test, and analysis of covariance (ANCOVA) were used and also, for non-normal variables the non-parametric Mann-Whitney $U$ test and Wilcoxon signed-rank test were employed. Binary logistic regression was also used to estimate the odds ratio (OR) of death. Survival analysis was conducted using the Kaplan-Meier curve and Cox regression.

\section{Results}

A total of 120 patients were recruited to the study. Patients were all confirmed with COVID-19 and hospitalized to ICU department for receiving intensive care. Advanced pulmonary involvement and requirement to respiratory support via positive pressure non-invasive ventilation was reported for all patients. Among included participants, 68 patients (56.7\%) were male and 52 patients (43.3\%) were female. Mean age was $60.29 \pm 12.94$ years with a range of $29-97$ years (Table 1). Co-existing conditions, like cardiovascular disease, diabetes, or chronic respiratory disease was seen for 84 patients (70\%) (Table 1). 60 patients were randomly assigned to the medication group and an equal number assigned to the placebo group. None of the patients received any vaccine before and during the trial. All patients completed the study, either recovered or died, and no participant left the trial (figure 1). Table 1 shows some baseline information for both groups at day 0 . Groups demonstrated homogenous assignment for majority of parameters, however, despite random assignment age mean was different between groups. Therefore, in order to adjust the age effect as a confounding variable we used analysis of covariance (ANCOVA) test to compare the groups on the 7th day.

\section{Primary Outcomes}

\section{Mortality}

Until day 14 , death occurred in five patients (8.33\%) in the antiviral preparation group, while in 36 patients (60\%) of the control group, which means highly significant difference in mortality between medication and placebo groups. The probability of death for patients received the medication was 0.14 times of those for the placebo group (risk ratio: $0.14 ; 95 \%$ confidence interval [ $\mathrm{Cl}], 0.05$ to $0.32 ; \mathrm{P}<0.001$ ). To determine the odds ratio of mortality, logistic regression analysis was conducted. The results demonstrated that the age-adjusted odds ratio of death for the placebo group compared to the antiviral preparation group was 14.63 (95\% confidence interval [Cl], 4.95 to $43.24 ; \mathrm{P}<0.001)$. We also evaluated the effect of the intervention on median survival during 14 days as age-adjusted hazard ratio derived from Cox regression was 0.12 (95\% confidence interval [CI], 0.04 to 0.31 ; $\mathrm{P}<0.001)$. The Kaplan-Meier curve along with Log Rang test also showed significant difference in the survival rate between both groups (figure 2). In fact, the patients received the antiviral preparation were predicted with higher survival compared to the placebo group.

\section{Secondary Outcomes}

Due to low sample size of the placebo group resulting from missed dead patients on the $14^{\text {th }}$ day, improvement in several clinical and laboratory parameters of patients was evaluated through day 7 (Table 2). Some respiratory parameters like respiratory rate (RR) showed significant improvement in patients treated with the antiviral preparation on day 7 compared to the baseline measurement; however, the change was not significant compared to the placebo group. Several laboratory parameters like peripheral blood lymphocyte and neutrophil count, and total bilirubin showed improvement in the antiviral preparationtreated patients, while lymphocyte count and serum glutamic pyruvic transaminase (SGPT) were exacerbated in the placebo group. Overall, white blood cells (WBCs) count, CRP, and calcium were improved in antiviral preparation-treated patients compared to the placebo group. Importantly, $\mathrm{SpO}_{2}$ demonstrated highly significant improvement in the treatment compared with the placebo group. No adverse effect was reported for patients in association with the treatment.

\section{Discussion}

The world has been facing the COVID-19 pandemic since late 2019, which has affected millions of lives and threatened billion others. Little is known about the pathophysiological details of SARS-CoV-2 virus, while it affects both genders, a wide age range (although with lower pathogenesis in children compared to more severe disease in the elderly), races, and particularly individuals with comorbidities like diabetes, hypertension, and underlying pulmonary diseases in all continents. The majority of COVID-19 cases are asymptomatic, recover from mild illness spontaneously or after receiving supportive therapies. However, millions of deaths have resulted from severe pulmonary involvement contributing to multi-organ failure. The highest rates of mortality among hospitalized patients (up to $88 \%$ ) have been reported in ICU-admitted cases, which require intensive pulmonary support due to advanced lung involvement and comorbidities (17).

Taking less than one year, SARS-CoV-2 vaccines have been developed most quickly compared to any other vaccine in the history (18). To date, several vaccines have received emergency or final approval for clinical use, a number of others are in the pipeline, and nearly 4 billion people (more than half of the global population) have received at least one dose worldwide (19). Globally, these vaccines have shown robust effects in the prevention of severe disease and subsequently reduced COVID-19-associated mortality, particularly among older and susceptible individuals (20-22). However, new SARS-CoV-2 infections, although mainly milder, have been reported to occur in a number of vaccinated people (23). Moreover, to achieve high protection in a society, the majority of the population are required to get vaccinated since unvaccinated individuals may drive further outbreaks (24). This may take more extended time, investment, and comprehensive policies to support mass vaccinations in addition to requirements for global equal distribution. Additionally, high effectiveness of SARSCoV-2 vaccines against novel viral variants resulted from frequent mutations is a place of doubt and requires further investigations and optimizations (25). 
Due to lack of definite preventive or therapeutic approaches, conventional interventions like face masks and social distancing are still recommended as nonpharmacological interventions particularly in settings with high possibility of transmission in addition to vaccination for breaking the virus transmission cycle until its eradication (26).

Hitherto, no efficient therapy has been developed for COVID-19 patients and several suggested treatments like convalescent plasma taken from already infected patients, and repurposed drugs like chloroquine, hydroxychloroquine, ribavirin, lopinavir-ritonavir, favipiravir, and ivermectin have failed in several clinical trials to demonstrate significant impact on the course of the disease (27-29). Although studies have shown reduced hospitalization, recovery time and mortality with the use of a number of drugs like the antiviral remdesivir (during the early phase of disease), interferons, and dexamethasone (30); these agents could not be considered as definite treatments for COVID-19 as their efficacies in other studies have been questioned and several drug agencies have not approved their clinical use unlike others. Additionally, although mass vaccinations have helped improved management of patients preventing severe illness, still a fraction of patients are admitted to take intensive support, among them mortality happens particularly in patients with underlying diseases.

In this randomized, double-blind, placebo-controlled phase III trial, we showed the efficacy and safety of a novel antiviral preparation in ICU-hospitalized patients with COVID-19. In our previous study, we showed that the antiviral preparation inhibited viral load of RNA viruses (data not published yet). Our results demonstrated $91.67 \%$ recovery of patients after up to two weeks of treatment, and $8.33 \%$ mortality rate in the antiviral preparation-treated patients compared with $40 \%$ recovery and $60 \%$ mortality in the placebo group. These numbers unveiled a highly significant impact of the antiviral preparation in reducing COVID19-associated deaths. COVID-19 patients in the control group, who received normal saline on top of standard care, had age-adjusted odds ratio (OR) of death of about 14.63 compared with those given the antiviral preparation. Survival analysis also demonstrated a significantly higher survival rate in the antiviral preparation-treated versus control patients. Additionally, clinical and laboratory parameters demonstrated improvements in the treatment group after 7 days compared with placebo-prescribed patients. The observed efficacy could be attributed to the activity of the herbal ingredients of the preparation against various types of human viral pathogens like hepatitis viruses, attenuated human immunodeficiency virus type 1 (HIV-1), influenza virus, and SARS-CoV (3133). For instance, Glycyrrhiza glabra and its phytochemicals seem to inhibit the viral replication, and also affect the intracellular signaling, gene expression, inducible nitric oxide synthase, and nitric oxide production in the macrophages (34). Although vaccination is still the best form of prevention, many countries (particularly some in Africa) still have a vaccination rate of less than $10 \%$ (https://ourworldindata.org/covid-vaccinations). It is suggested that the use of this natural mixture could aid recovery in such poorly vaccinated countries and territories. In the UK, which has fully vaccinated more than $80 \%$ of individuals over 18 years old, National Health Service statistics has shown that non-vaccinated subjects have a 32-fold greater risk of death from COVID-19 compared to fully vaccinated individuals (35). The novel preparation introduced in this study may also improve outcomes in those who have been fully vaccinated but this will have to be tested in future trials.

\section{Conclusion}

The herbal antiviral preparation demonstrated high efficacy in the treatment of critically ill ICU-admitted patients with COVID-19. It significantly reduced mortality rate, and improved clinical and laboratory parameters compared to the placebo group. Our results demonstrated good efficacy and safety of the preparation surpassing other suggested treatments. Taken together, the novel antiviral preparation can be considered as a potential therapeutic agent for SARS-CoV-2-infected patients, not only for critically ill patients but also for outpatients. Potentially the greatest impact on the use of this preparation will be seen in the likely advent of future pandemics before new vaccines can be produced and deployed around the world. Further investigations are recommended to discover the therapeutic effects of the antiviral preparation in different subsets of COVID-19 patients along with mechanistic pharmacological studies.

\section{Declarations}

\section{Declaration of Competing Interest}

Ahmad Hosseinpour is the founder and director of Shimi Teb Salamat Co.

\section{Acknowledgment}

The authors acknowledge the health workers and officials at Ali Asghar Hospital as well as our colleagues at Shiraz Food and Drug Administration, Health Policy Research Center, Ethical Committee, Deputy of Treatment and Chancellor of Shiraz University of Medical Sciences who enabled us to perform successfully the study.

\section{Consent for publication}

The results will be used for research purposes and will be published.

\section{Funding}

This study was supported in full by Shimi Teb Salamat Co., Shiraz, Iran.

\section{Availability of supporting data}

In case of requesting data for the study, the applicant must first introduce himself or herself and the relevant organization to determine the purpose of the data request and state for what purpose this data is used. After submitting the request, if the researchers of this study prove that the data of this study can advance the therapeutic goals, the information will be sent as long as the data remains confidential. The data are available upon request to the corresponding author.

\section{Authors' contributions}


AH designed the study, HF, AS, MRH,VK, SN, FF, and AM performed the experiments and wrote the manuscript, FC performed the statistical analyses, AH supervised the study. All authors have contributed to the study, and read and approved the submitted manuscript.

\section{Ethical Approval and Consent to participate}

The research was approved by the Ethics Committee of the Shiraz University of Medical Sciences (Ethics committee reference number: IR.SUMS.REC.1399.1367. Approval date 15March2021). Consent of participation in the research project has been obtained from all patients.

\section{References}

1. Wang C, Horby PW, Hayden FG, Gao GF. A novel coronavirus outbreak of global health concern. The Lancet. 2020;395(10223):470-3.

2. Organization WH. WHO Coronavirus (COVID-19) Dashboard 2021. Available from: https://covid19.who.int/.

3. V'kovski P, Kratzel A, Steiner S, Stalder H, Thiel V. Coronavirus biology and replication: implications for SARS-CoV-2. Nature Reviews Microbiology. 2021;19(3):155-70.

4. Hu S, Wang W, Wang Y, Litvinova M, Luo K, Ren L, et al. Infectivity, susceptibility, and risk factors associated with SARS-CoV-2 transmission under intensive contact tracing in Hunan, China. Nature Communications. 2021;12(1):1533.

5. Piroth L, Cottenet J, Mariet A-S, Bonniaud P, Blot M, Tubert-Bitter P, et al. Comparison of the characteristics, morbidity, and mortality of COVID-19 and seasonal influenza: a nationwide, population-based retrospective cohort study. The Lancet Respiratory Medicine. 2021;9(3):251-9.

6. Myers LC, Parodi SM, Escobar GJ, Liu VX. Characteristics of Hospitalized Adults With COVID-19 in an Integrated Health Care System in California. JAMA. 2020;323(21):2195-8.

7. Arentz M, Yim E, Klaff L, Lokhandwala S, Riedo FX, Chong M, et al. Characteristics and Outcomes of 21 Critically III Patients With COVID-19 in Washington State. JAMA. 2020;323(16):1612-4.

8. Oliveira E, Parikh A, Lopez-Ruiz A, Carrilo M, Goldberg J, Cearras M, et al. ICU outcomes and survival in patients with severe COVID-19 in the largest health care system in central Florida. PLOS ONE. 2021;16(3):e0249038.

9. Elezkurtaj S, Greuel S, Ihlow J, Michaelis EG, Bischoff P, Kunze CA, et al. Causes of death and comorbidities in hospitalized patients with COVID-19. Scientific Reports. 2021;11(1):4263.

10. Cook TM, Roberts JV. Impact of vaccination by priority group on UK deaths, hospital admissions and intensive care admissions from COVID-19. Anaesthesia. 2021;76(5):608-16.

11. Alencar CH, Cavalcanti LPdG, Almeida MMd, Barbosa PPL, Cavalcante KKdS, Melo DNd, et al. High Effectiveness of SARS-CoV-2 Vaccines in Reducing COVID-19-Related Deaths in over 75-Year-Olds, Ceará State, Brazil. Tropical medicine and infectious disease. 2021;6(3):129.

12. Moghadas SM, Vilches TN, Zhang K, Wells CR, Shoukat A, Singer BH, et al. The impact of vaccination on COVID-19 outbreaks in the United States. Clin Infect Dis. 2021:ciab079.

13. Vilches TN, Zhang K, Van Exan R, Langley JM, Moghadas SM. Projecting the impact of a two-dose COVID-19 vaccination campaign in Ontario, Canada. Vaccine. 2021;39(17):2360-5.

14. Albani F, Fusina F, Granato E, Capotosto C, Ceracchi C, Gargaruti R, et al. Corticosteroid treatment has no effect on hospital mortality in COVID-19 patients. Scientific Reports. 2021;11(1):1015.

15. Reis G, Moreira Silva EAdS, Medeiros Silva DC, Thabane L, Singh G, Park JJH, et al. Effect of Early Treatment With Hydroxychloroquine or Lopinavir and Ritonavir on Risk of Hospitalization Among Patients With COVID-19: The TOGETHER Randomized Clinical Trial. JAMA Network Open. 2021;4(4):e216468e.

16. Hoek JM, Field SM, de Vries YA, Linde M, Pittelkow M-M, Muradchanian J, et al. Rethinking remdesivir for COVID-19: A Bayesian reanalysis of trial findings. PLOS ONE. 2021;16(7):e0255093.

17. Rieg S, von Cube M, Kalbhenn J, Utzolino S, Pernice K, Bechet L, et al. COVID-19 in-hospital mortality and mode of death in a dynamic and non-restricted tertiary care model in Germany. PloS one. 2020;15(11):e0242127-e.

18. Funk CD, Laferrière C, Ardakani A. A Snapshot of the Global Race for Vaccines Targeting SARS-CoV-2 and the COVID-19 Pandemic. Frontiers in pharmacology. 2020;11:937.

19. Times NY. Tracking Coronavirus Vaccinations Around the World 2021 [Sep. 08, 2021]. Available from: https://www.nytimes.com/interactive/2021/world/covid-vaccinations-tracker.html.

20. Lopez Bernal J, Andrews N, Gower C, Robertson C, Stowe J, Tessier E, et al. Effectiveness of the Pfizer-BioNTech and Oxford-AstraZeneca vaccines on covid-19 related symptoms, hospital admissions, and mortality in older adults in England: test negative case-control study. BMJ. 2021;373:n1088.

21. Dagan N, Barda N, Kepten E, Miron O, Perchik S, Katz MA, et al. BNT162b2 mRNA Covid-19 Vaccine in a Nationwide Mass Vaccination Setting. New England Journal of Medicine. 2021;384(15):1412-23.

22. Tregoning JS, Flight KE, Higham SL, Wang Z, Pierce BF. Progress of the COVID-19 vaccine effort: viruses, vaccines and variants versus efficacy, effectiveness and escape. Nature Reviews Immunology. 2021.

23. Bergwerk M, Gonen T, Lustig Y, Amit S, Lipsitch M, Cohen C, et al. Covid-19 Breakthrough Infections in Vaccinated Health Care Workers. New England Journal of Medicine. 2021.

24. Dhama K, Sharun K, Tiwari R, Dhawan M, Emran TB, Rabaan AA, et al. COVID-19 vaccine hesitancy - reasons and solutions to achieve a successful global vaccination campaign to tackle the ongoing pandemic. Human Vaccines \& Immunotherapeutics. 2021:1-5.

Page 6/12 
25. Madhi SA, Baillie V, Cutland CL, Voysey M, Koen AL, Fairlie L, et al. Efficacy of the ChAdOx1 nCoV-19 Covid-19 Vaccine against the B.1.351 Variant. New England Journal of Medicine. 2021;384(20):1885-98.

26. Rowan NJ, Moral RA. Disposable face masks and reusable face coverings as non-pharmaceutical interventions (NPIs) to prevent transmission of SARSCoV-2 variants that cause coronavirus disease (COVID-19): Role of new sustainable NPI design innovations and predictive mathematical modelling. Science of The Total Environment. 2021;772:145530.

27. Martinez MA. Lack of Effectiveness of Repurposed Drugs for COVID-19 Treatment. Frontiers in Immunology. 2021;12(653).

28. Agarwal A, Mukherjee A, Kumar G, Chatterjee P, Bhatnagar T, Malhotra P. Convalescent plasma in the management of moderate covid-19 in adults in India: open label phase II multicentre randomised controlled trial (PLACID Trial). BMJ. 2020;371:m3939.

29. López-Medina E, López P, Hurtado IC, Dávalos DM, Ramirez O, Martínez E, et al. Effect of Ivermectin on Time to Resolution of Symptoms Among Adults With Mild COVID-19: A Randomized Clinical Trial. JAMA. 2021;325(14):1426-35.

30. Welte T, Ambrose LJ, Sibbring GC, Sheikh S, Müllerová H, Sabir I. Current evidence for COVID-19 therapies: a systematic literature review. European Respiratory Review. 2021;30(159):200384.

31. Sato H, Goto W, Yamamura J, Kurokawa M, Kageyama S, Takahara T, et al. Therapeutic basis of glycyrrhizin on chronic hepatitis B. Antiviral research. 1996;30(2-3):171-7.

32. Ito M, Nakashima H, Baba M, Pauwels R, De Clercq E, Shigeta S, et al. Inhibitory effect of glycyrrhizin on the in vitro infectivity and cytopathic activity of the human immunodeficiency virus [HIV (HTLV-III/LAV)]. Antiviral research. 1987;7(3):127-37.

33. Utsunomiya T, Kobayashi M, Pollard RB, Suzuki F. Glycyrrhizin, an active component of licorice roots, reduces morbidity and mortality of mice infected with lethal doses of influenza virus. Antimicrobial agents and chemotherapy. 1997;41(3):551-6.

34. Nassiri Asl M, Hosseinzadeh H. Review of Antiviral Effects of Glycyrrhiza glabra L. and Its Active Component, Glycyrrhizin. jmpir. 2007;6(22):1-12.

35. https://www.ons.gov.uk/peoplepopulationandcommunity/birthsdeathsandmarriages/deaths/bulletins/deathsinvolvingcovid19byvaccinationstatusenglar fbclid=IwAR1E3rCbwvY3a7SqMbwJhSGvdSNdQboUcSGYdXMCzzkHMO-mXatxz4Wvxgc.

\section{Tables}

Table 1. Baseline clinical and laboratory values for medication and placebo groups. 
Qualitative variables

\begin{tabular}{|c|c|c|c|c|c|}
\hline Variables & & Medication $(n=60)$ & Placebo $(n=60)$ & Total $(n=120)$ & $p$-value ${ }^{a}$ \\
\hline \multirow[t]{3}{*}{ Age group } & $<45$ & $10(17.2)$ & $4(6.8)$ & $14(11.7)$ & \multirow{3}{*}{0.029} \\
\hline & $45-64$ & 33 (56.9) & $27(45.8)$ & $60(50)$ & \\
\hline & $\geq 65$ & $15(25.9)$ & $28(47.5)$ & $43(35.8)$ & \\
\hline \multirow[t]{2}{*}{$\operatorname{Sex}(\%)$} & Male & $32(53.3)$ & $36(60)$ & $68(56.7)$ & \multirow[t]{2}{*}{0.46} \\
\hline & Female & $28(46.7)$ & $24(40)$ & $52(43.3)$ & \\
\hline \multirow[t]{2}{*}{ Disease history (\%) } & Yes & $41(68.3)$ & $43(71.7)$ & $84(70)$ & \multirow[t]{2}{*}{0.69} \\
\hline & No & $19(31.7)$ & $17(28.3)$ & $36(30)$ & \\
\hline \multirow[t]{7}{*}{ Co-existing Diseases } & Cardiovascular disease & $18(30)$ & $19(31.7)$ & $37(30.8)$ & \multirow{6}{*}{0.88} \\
\hline & Diabetes & $11(18.3)$ & $9(15)$ & $20(16.7)$ & \\
\hline & Respiratory disease & $3(5)$ & $4(4)$ & $7(5.8)$ & \\
\hline & Endocrine disease & $4(6.7)$ & $2(3.3)$ & $6(5)$ & \\
\hline & Transplantation & $3(5)$ & $1(1.7)$ & $4(3.3)$ & \\
\hline & Other & $4(6.7)$ & $6(10)$ & $10(8.3)$ & \\
\hline & None & $17(28.3)$ & $19(31.7)$ & $36(30)$ & - \\
\hline \multirow[t]{2}{*}{ RT-PCR positive results } & Yes & 59 (98.3) & $53(88.3)$ & $112(93.3)$ & \multirow[t]{2}{*}{0.06} \\
\hline & No & $1(1.7)$ & $7(11.7)$ & $8(6.7)$ & \\
\hline \multicolumn{6}{|l|}{ Quantitative variables } \\
\hline & & \multirow[t]{2}{*}{ Case $(n=60)$} & \multirow[t]{2}{*}{ Control $(n=60)$} & Total & \multirow[t]{2}{*}{ P-value ${ }^{b}$} \\
\hline & & & & Mean \pm SD & \\
\hline \multicolumn{2}{|l|}{ Age } & $56.78 \pm 12.31$ & $63.75 \pm 12.71$ & $60.29 \pm 12.94$ & 0.003 \\
\hline \multicolumn{2}{|l|}{ BMI } & $27.31 \pm 3.95$ & $25.87 \pm 5.82$ & $26.59 \pm 5$ & 0.12 \\
\hline \multicolumn{2}{|l|}{ HR } & $81.88 \pm 15.85$ & $83.44 \pm 19.62$ & $82.65 \pm 17.74$ & 0.63 \\
\hline \multicolumn{2}{|l|}{ Body Temperature } & $36.74 \pm 0.27$ & $36.75 \pm 0.39$ & $36.74 \pm 0.33$ & 0.79 \\
\hline \multicolumn{2}{|l|}{ RR } & $24.68 \pm 3.98$ & $26.49 \pm 13.35$ & $25.56 \pm 9.74$ & 0.07 \\
\hline \multicolumn{2}{|l|}{ WBC } & $11.7 \pm 7.39$ & $13.2 \pm 7.52$ & $12.46 \pm 7.46$ & 0.27 \\
\hline \multicolumn{2}{|l|}{ Systolic BP } & $123.73 \pm 20.43$ & $118.66 \pm 19.03$ & $121.2 \pm 19.82$ & 0.16 \\
\hline \multicolumn{2}{|l|}{ Diastolic BP } & $73.51 \pm 16.46$ & $74.06 \pm 20.22$ & $73.79 \pm 18.36$ & 0.87 \\
\hline \multicolumn{2}{|l|}{ LYM } & $6.4(5.1,9)$ & $6.65(4.22,9.37)$ & $6.6(4.75,9.05)$ & 0.34 \\
\hline \multicolumn{2}{|l|}{ NEUT } & $86.45 \pm 6.6$ & $87.21 \pm 6.52$ & $86.81 \pm 7.66$ & 0.66 \\
\hline \multicolumn{2}{|l|}{ Serum Creatinine } & $1.1(0.9,1.3)$ & $1.2(0.9,1.7)$ & $1.1(0.9,1.5)$ & 0.11 \\
\hline $\mathrm{SPO}_{2}$ & & $89.68 \pm 7.69$ & $86.66 \pm 10.07$ & $88.19 \pm 9.03$ & 0.07 \\
\hline SGOT & & $44(34,55)$ & $39(25,71)$ & $40.5(27.75,62.5)$ & 0.76 \\
\hline SGPT & & $42(30.75,70.5)$ & $39(28,68)$ & $40(30,68.5)$ & 0.55 \\
\hline $\mathrm{Hb}$ & & $13.84 \pm 2.25$ & $13.76 \pm 2.46$ & $13.8 \pm 2.35$ & 0.85 \\
\hline pH & & $7.32 \pm .78$ & $7.36 \pm 0.1$ & $7.34 \pm 0.55$ & 0.69 \\
\hline $\mathrm{PCO}_{2}$ & & $41.42 \pm 9.76$ & $42.99 \pm 11.93$ & $42.21 \pm 10.88$ & 0.43 \\
\hline Total Bilirubin & & $.84 \pm .44$ & $1.24 \pm 1.83$ & $0.89 \pm 0.5$ & 0.21 \\
\hline INR & & $1.24 \pm .28$ & $1.22 \pm 0.3$ & $1.23 \pm 0.29$ & 0.72 \\
\hline CRP $(\mu \mathrm{g} / \mathrm{mL})$ & & $51.68 \pm 27.18$ & $54.48 \pm 26.97$ & $52.83 \pm 26.99$ & 0.61 \\
\hline LDH (U/L) & & $1096.8 \pm 393.05$ & $1199 \pm 730.33$ & $1144.63 \pm 574.32$ & 0.36 \\
\hline Pulmonary involvement & & $68.53 \pm 9.6$ & $70.37 \pm 8.87$ & $69.07 \pm 9.37$ & 0.42 \\
\hline
\end{tabular}




\begin{tabular}{lllll} 
Sodium & $137.7 \pm 3.98$ & $137.9 \pm 8,72$ & $137.8 \pm 6.75$ & 0.87 \\
\hline Potassium & $4.13 \pm 0.49$ & $4.31 \pm ., 85$ & $4.22 \pm 0.69$ & 0.16 \\
\hline Calcium & $8.36 \pm 74$ & $8.08 \vee 1.15$ & $8.22 \pm 0.97$ & 0.13 \\
\hline Phosphorus & $3.29 \pm 0.79$ & $3.5 \pm 1.52$ & $3.38 \pm 1.16$ & 0.41 \\
\hline Magnesium & $2.43 \pm .38$ & $2.49 \pm .87$ & $2.42 \pm 0.42$ & 0.65
\end{tabular}

Note: Data are mean $\pm \mathrm{SD}$, median (IQR), or $\mathrm{N}(\%)$ as indicated

a Obtained from Chi-square or Fisher exact test

${ }^{b}$ Obtained from independent samples $t$ test (for parametric data) or Mann-Whitney Utest (for nonparametric data)

BMI: body mass index, HR: heart rate, RR: respiratory rate, WBC: white blood cells, LYM: lymphocyte, NEUT: neutrophile, BP: blood pressure, INR: international normalized ratio, SGOT: serum Glutamic-oxalacetic transaminase, SGPT: serum glutamic-pyruvic transaminase (SGPT), LDH: lactate dehydrogenase, Hb: hemoglobin

Table 2. Clinical and laboratory petameters post intervention. 


\begin{tabular}{|c|c|c|c|c|c|}
\hline Variables & groups & Day 0 & Day 7 & $\begin{array}{l}\text { p-value a } \\
\text { (Within group) }\end{array}$ & $\begin{array}{l}\text { p-value }{ }^{b} \\
\text { (Between group) }\end{array}$ \\
\hline \multirow[t]{2}{*}{ HR } & Medication & $82.03 \pm 17.84$ & $85.2 \pm 13.84$ & 0.28 & \multirow[t]{2}{*}{0.05} \\
\hline & Placebo & $87.44 \pm 16.45$ & $93.32 \pm 13.67$ & 0.13 & \\
\hline \multirow[t]{2}{*}{ Body Temperature } & Medication & $36.71 \pm 0.28$ & $36.7 \pm 0.32$ & 0.86 & \multirow[t]{2}{*}{0.32} \\
\hline & Placebo & $36.75 \pm 0.24$ & $36.8 \pm 0.36$ & 0.41 & \\
\hline \multirow[t]{2}{*}{ RR } & Medication & $24.93 \pm 3.55$ & $22.71 \pm 3.26$ & 0.001 & \multirow[t]{2}{*}{0.53} \\
\hline & Placebo & $23.8 \pm 5.49$ & $23.07 \pm 2.93$ & 0.52 & \\
\hline \multirow[t]{2}{*}{ WBC } & Medication & $12.4 \pm 9.49$ & $12.2 \pm 4.84$ & 0.92 & \multirow[t]{2}{*}{0.05} \\
\hline & Placebo & $12.45 \pm 6.33$ & $15.56 \pm 7.55$ & 0.08 & \\
\hline \multirow[t]{2}{*}{ Systolic BP } & Medication & $125.69 \pm 21.54$ & $124.54 \pm 15.48$ & 0.66 & \multirow[t]{2}{*}{0.18} \\
\hline & Placebo & $118.48 \pm 18.77$ & $116.76 \pm 14.75$ & 0.69 & \\
\hline \multirow[t]{2}{*}{ Diastolic BP } & Medication & $74.21 \pm 12.83$ & $75.03 \pm 11.41$ & 0.73 & \multirow[t]{2}{*}{0.69} \\
\hline & Placebo & $77.11 \pm 28.75$ & $73.64 \pm 13.7$ & 0.56 & \\
\hline \multirow[t]{4}{*}{ LYM } & Medication & $9.12 \pm 10.31$ & $12.49 \pm 12.14$ & 0.007 & \multirow[t]{4}{*}{0.31} \\
\hline & & $6.15(5.15,9)$ & $8.7(5.95,16.37)$ & & \\
\hline & Placebo & $7.14 \pm 4.01$ & $8.9 \pm 5.11$ & 0.04 & \\
\hline & & $5.6(4.6,9)$ & $7.1(5.5,12.85)$ & & \\
\hline \multirow[t]{2}{*}{ NEUT } & Medication & $85.64 \pm 10.95$ & $79.42 \pm 13.69$ & 0.003 & \multirow[t]{2}{*}{0.28} \\
\hline & Placebo & $87.9 \pm 5.42$ & $84.51 \pm 7.73$ & 0.03 & \\
\hline \multirow[t]{2}{*}{ Serum Creatinine } & Medication & $1.1(0.9,1.4)$ & $1.2(0.9,1.4)$ & 0.93 & \multirow[t]{2}{*}{0.58} \\
\hline & Placebo & $1.2(0.9,1.45)$ & $1.05(0.8,1.3)$ & 0.21 & \\
\hline \multirow[t]{2}{*}{$\mathrm{SpO}_{2}$} & Medication & $89.68 \pm 7.69$ & $92.7 \pm 3.39$ & 0.005 & \multirow[t]{2}{*}{$<0.001$} \\
\hline & Placebo & $87.54 \pm 10.23$ & $83.74 \pm 13.49$ & 0.07 & \\
\hline \multirow[t]{2}{*}{ SGOT } & Medication & $44(34,55)$ & $43(30,54)$ & 0.35 & \multirow[t]{2}{*}{0.32} \\
\hline & Placebo & $38.5(24.75,65)$ & $39(23.75,90)$ & 0.3 & \\
\hline \multirow[t]{2}{*}{ SGPT } & Medication & $37(29.75,58.25)$ & $57(35.25,75)$ & 0.09 & \multirow[t]{2}{*}{0.82} \\
\hline & Placebo & $36.5(29.25,76)$ & $60.5(42,89.5)$ & 0.03 & \\
\hline \multirow[t]{2}{*}{$\mathrm{Hb}$} & Medication & $13.52 \pm 2.4$ & $13.38 \pm 2.05$ & 0.61 & 0.32 \\
\hline & Placebo & $14 \pm 2.46$ & $13.28 \pm 2.88$ & 0.1 & \\
\hline $\mathrm{pH}$ & Medication & $7.22 \pm 1.06$ & $7.23 \pm 1.06$ & 0.6 & 0.07 \\
\hline & Placebo & $7.37 \pm 0.1$ & $7.15 \pm 0.78$ & 0.12 & \\
\hline $\mathrm{PCO}_{2}$ & Medication & $41.44 \pm 10.71$ & $43 \pm 9.21$ & 0.36 & 0.44 \\
\hline & Placebo & $40.75 \pm 9.3$ & $45.88 \pm 14.02$ & 0.06 & \\
\hline Total Bilirubin & Medication & $0.9 \pm 0.55$ & $1.16 \pm 0.7$ & 0.02 & 0.81 \\
\hline & Placebo & $0.87 \pm 0.33$ & $1.1 \pm 0.42$ & 0.08 & \\
\hline INR & Medication & $1.23 \pm 0.23$ & $1.14 \pm 0.23$ & 0.1 & 0.17 \\
\hline & Placebo & $1.15 \pm 0.18$ & $1.22 \pm 0.29$ & 0.29 & \\
\hline CRP & Medication & $53(27,72.75)$ & $15(5.5,37)$ & $<0.001$ & 0.01 \\
\hline & Placebo & $58(50,73)$ & $60(23,76)$ & 0.47 & \\
\hline LDH & Medication & $1161.73 \pm 440.7$ & $1015.56 \pm 451.82$ & 0.06 & 0.99 \\
\hline & Placebo & $1129.76 \pm 447.36$ & $999.82 \pm 447.36$ & 0.44 & \\
\hline Sodium & Medication & $137.6 \pm 4.19$ & $137.18 \pm 3.8$ & 0.62 & 0.83 \\
\hline & Placebo & $139.27 \pm 12.39$ & $138.15 \pm 6.39$ & 0.58 & \\
\hline
\end{tabular}




\begin{tabular}{llllll} 
Potassium & Medication & $4.21 \pm 0.53$ & $4.27 \pm 0.48$ & 0.61 & 0.42 \\
\cline { 2 - 5 } Calcium & Placebo & $4.28 \pm 0.81$ & $4.11 \pm 0.68$ & 0.49 & \\
& Medication & $8.18 \pm 0.83$ & $8.59 \pm 0.65$ & 0.02 & 0.031 \\
\cline { 2 - 5 } Phosphorus & Placebo & $8.08 \pm 0.99$ & $8.25 \pm 0.75$ & 0.53 & \\
& Medication & $3.44 \pm 0.83$ & $3.52 \pm 1.15$ & 0.74 & 0.81 \\
\cline { 2 - 5 } Magnesium & Placebo & $3.39 \pm 1.48$ & $3.32 \pm 0.99$ & 0.82 & \\
& Medication & $2.36 \pm 0.33$ & $2.31 \pm 0.28$ & 0.44 & 0.54
\end{tabular}

Note: Data are mean \pm SD or median (IQR) as indicated

a. Obtained from pair sample $t$ test (for parametric data) or Wilcoxon (for nonparametric data)

b: Obtained from Analysis of Covariance test, by adjusting age and baseline value

\section{Figures}

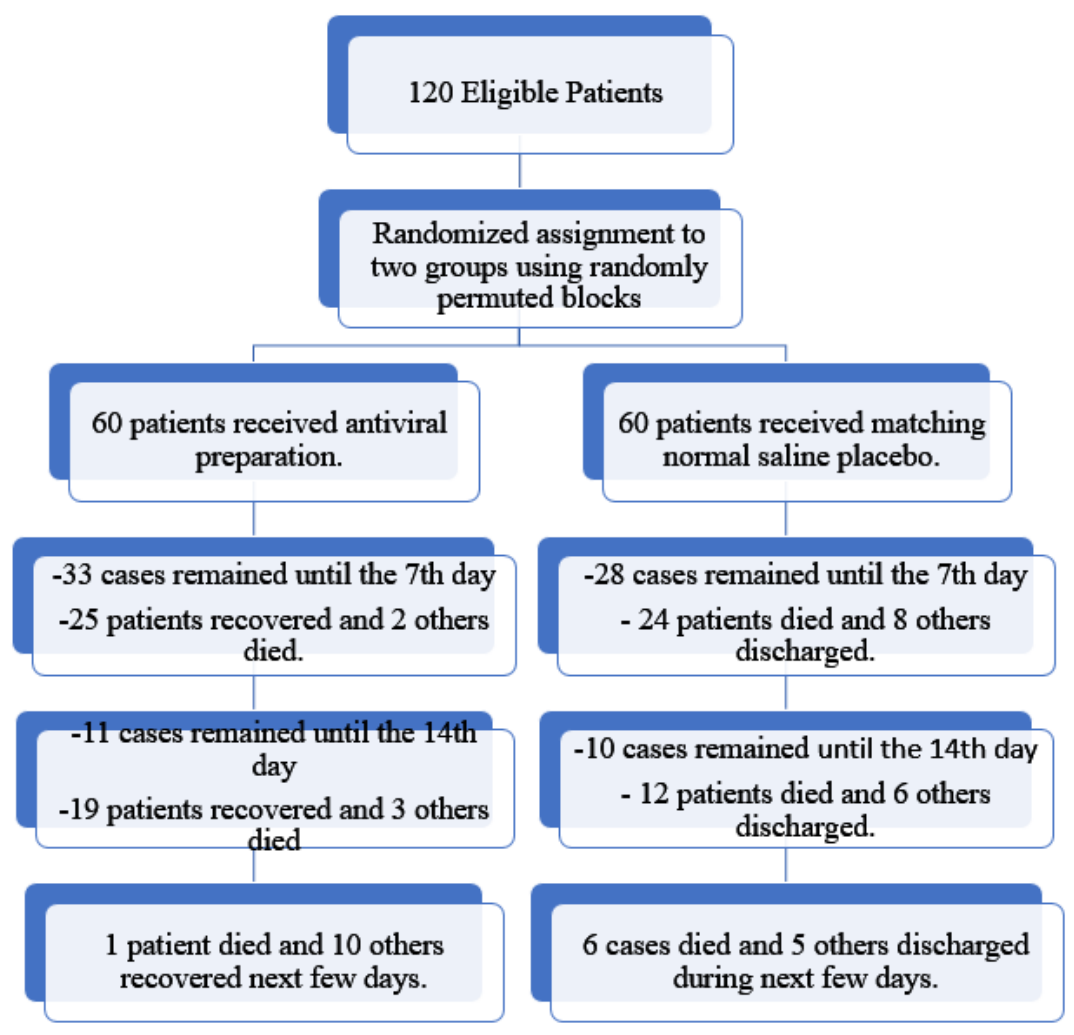

Figure 1

A total number of 120 ICU-admitted COVID-19 patients were recruited to the study and randomly assigned to two group of medication and intervention. The antiviral preparation -treated patients demonstrated lower deaths compared to the control group on days 7,14 , and post- 14 .

Flowchart of the study 


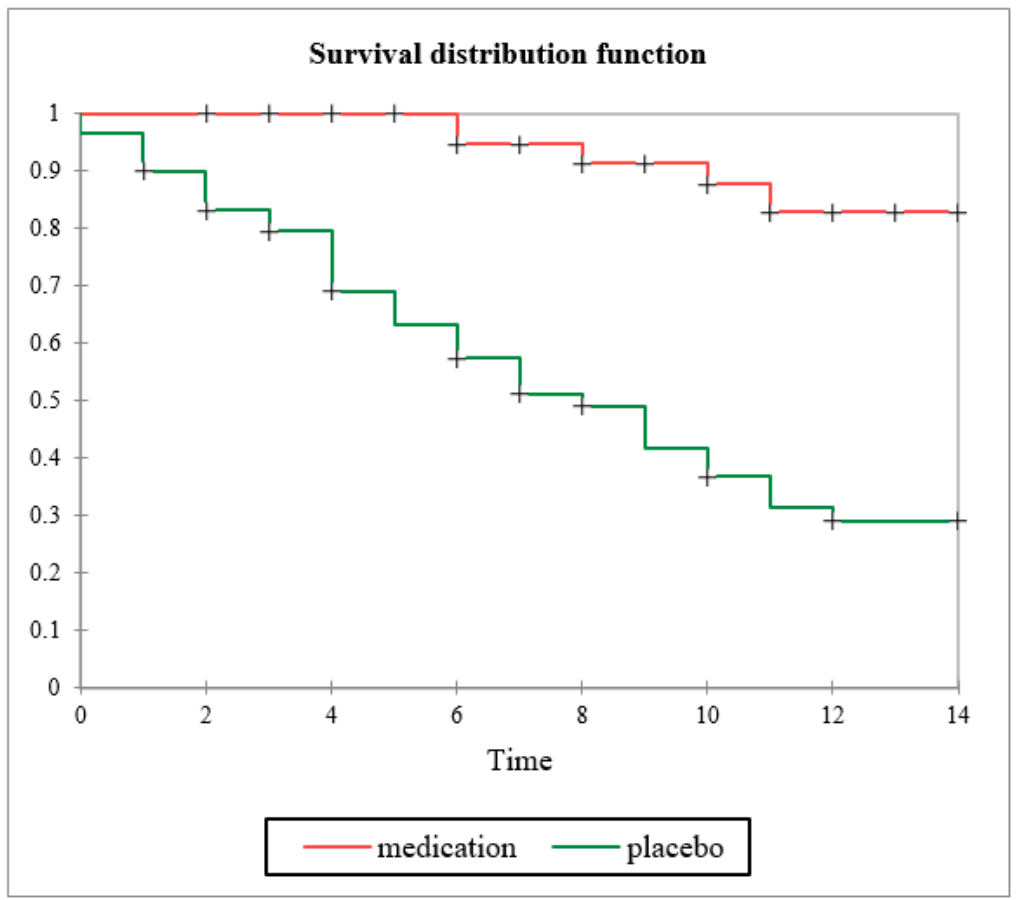

Figure 2

Survival analysis in Kaplan-Meier analysis shows decreased survival in placebo group compared to the control group

\section{Supplementary Files}

This is a list of supplementary files associated with this preprint. Click to download.

- 2178420unknownupload2228064r3rbfdsc.pdf

- 2178420unknownupload2228065r3rfffsc.pdf

- 2178420unknownupload2228063r3rtfb.pdf

- 2178420unknownupload2228068r3rjfm.pdf

- 2178420unknownupload2228069r3rhfq.pdf 